\title{
Закономерности адаптации систем к функциональным требованиям*
}

\author{
С. И. МАторин \\ ' ЗАО «СофтКоннект», г. Белгород, Россия \\ ' Национальный исследовательский университет «Белгородский государ- \\ ственный университет», г. Белгород, Россия
}

\begin{abstract}
Аннотация. В работе обсуждаются возможности анализа адаптации систем к функциональным требованиям с помощью системно-объектного подхода «Узел-Функция-Объект». Дано содержательное описание процесса адаптации в терминах системно-объектного подхода. Для формального описания процесса адаптации применен аппарат исчисления объектов Абади-Кардели. Показана связь адаптации с общесистемными закономерностями. Разработана схема пассивной адаптации самонастраивающихся систем, учитывающая данные закономерности.
\end{abstract}

Ключевые слова: системно-объектный подход «Узел-Функция-Объект», адаптация, внешняя детерминанта; внутренняя детерминанта; общесистемные закономерности.

DOI: $10.14357 / 20790279200105$

\section{Введение}

Адаптация (от лат. adaptare - приспособлять) в широком смысле способность системы приспосабливаться к изменяющимся условиям среды [1]. Данная способность обуславливает важнейшее свойство любой системы - ее выживаемость. Изучению процесса адаптации уделяется много внимания, в первую очередь, в биологии, медицине, социологии. Понятие «адаптация» стало широко фигурировать в математических и технических науках $[2,3]$. В системных исследованиях изучается адаптивная устойчивость систем. Рассматриваются адаптивные механизмы, которые имеют принципиально комбинаторную природу и представляют собой адаптивные цепочки преобразований, компенсирующих изменения окружающей среды [4].

В кибернетике адаптация - это процесс накопления и использования информации в системе, направленный на достижение определенного, обычно оптимального в некотором смысле, состояния или поведения системы при начальной неопределенности и изменяющихся внешних условиях. Рассматриваются адаптивные системы, которые при функционировании демонстрируют способность целенаправленно приспосабливаться к изменениям как внутренних, так и внешних условий. Различают

\footnotetext{
* Работа поддержана проектами РФФИ №18-07-00355a, №1807-00356а, №19-07-00290a, № 19-07-00111a, 19-29-01047мк, 16-29-12864офи-м.
}

пассивную адаптацию (реагирование системы на изменение окружающей среды) и активную адаптацию (воздействие системы на окружающую среду). Сами адаптивные системы подразделяются на самонастраивающиеся и самоорганизующиеся системы. В первом случае в соответствии с изменениями внешней среды меняются параметры функционирования системы, а во втором - меняется структура и организация системы [5].

Распространенность понятия «адаптация» в научной среде и использование этого термина специалистами различных предметных областей требуют рассмотрения адаптации как общесистемного явления. В данной статье пассивная адаптация самонастраивающихся систем к функциональным требованиям анализируется с помощью системно-объектного подхода «Узел-Функция-Объект» [6].

\section{1. Системно-объектный подход к процессу адаптации}

Исследуем содержательно процесс адаптации средствами системно-объектного подхода, рассматривающего систему как функциональный объект, функция которого обусловлена функцией объекта более высокого яруса (т.е. функцией надсистемы) [7].

В рамках данного подхода используется термин «адаптация системы к запросу надсистемы», обозначающий процесс приближения внутренней 
детерминанты системы к ее внешней детерминанте. При этом внешняя детерминанта системы (функциональный запрос надсистемы) представляет собой требуемую функцию системы, обусловленную функцией надсистемы; а внутренняя детерминанта системы - общая функция системы, т.е. ее фактическое функционирование. Последняя определяет функции подсистем (частные функции системы) и их взаимосвязи, т.е. внутренние субстанциальные, функциональные и структурные характеристики системы. Таким образом далее будет исследоваться пассивная адаптация самонастраивающихся систем.

Рассмотрим подробнее процесс формирования (становления) системы, так как при его анализе понятие «адаптация» проявляется достаточно полно $[7,8]$.

Предпосылкой начала процесса формирования системы, согласно системно-объектному подходу, является возникновение противоречия между функционированием надсистемы и поддерживающими надсистему функциями ее систем, т.е. противоречия между функциональными и поддерживающими потоками надсистемы. Это противоречие представляет собой нарушение баланса потоков связей (экстенциальных валентностей) в соответствующем узле надсистемы, когда возникают свободные интенциальные валентности окрестностных систем, а узел оказывается вакантным. Противоречие в виде интенциальных валентностей, порождающее формирование системы, представляет собой функциональный запрос надсистемы (т.е. внешнюю детерминанту системы). Этот запрос, представленный в виде вакантного узла надсистемы, определяет потребность надсистемы, т.е. появившуюся необходимость в системе с данной функцией. Таким образом, запрос (внешняя детерминанта) задает область требуемых функциональных состояний (ОТФС) для формирующейся системы через интенциальные валентности (связи) окрестностных систем.

В соответствии с внешней детерминантой системы, задающей область требуемых функциональных состояний, из резерва (набора систем) выбирается некоторая система как исходный материал. Эта система обладает областью возможных состояний (ОВС), характеризующей ее предрасположенность (интенцию/потенцию) к выполнению определенных (в данном случае требуемых надсистемой) функций. В результате фактического попадания исходного материала в вакантный узел надсистемы, необходимость превращается в возможность, потоки замыкаются, интенции превращаются в экстенции. Таким образом, система начинает функционировать в соответствии с запросом. При этом исходный материал превращается в субстанцию надсистемы, что и определяет процесс формирования системы с данными функциональными свойствами для поддержания функционирования надсистемы.

Фактическое функционирование системы в ранее вакантном узле надсистемы (новая внутренняя детерминанта системы) становится причиной возникновения противоречия между функциональными и поддерживающими потоками уже формируемой системы. Это противоречие становится причиной формирования подсистем с определенными функциями, поддерживающими систему, и т.д.

В результате описанного процесса система адаптируется к функциональному узлу надсистемы. Процесс адаптации системы к запросу надсистемы, таким образом, как заключительная фаза становления системы, начинается с того момента, когда данная система в качестве исходного материала помещается в соответствующий вакантный функциональный узел надсистемы. До начала адаптации, когда данная система еще является исходным материалом, внутренние поддерживающие свойства (потоки) данной системы имеют интенции (и скорее даже потенции) к поддержанию требуемых функциональных свойств (потоков), что и способствует выбору именно данной системы в качестве исходного материала. Но в то же самое время внутренние свойства (потоки) данной системы, как явления, потенциально и даже экстенциально могут и поддерживают множество других, в данном случае не требуемых, функциональных свойств. Это и обеспечивает ширину области возможных состояний системы (исходного материала), достаточную для включения области требуемых функциональных состояний вакантного узла, иными словами, определенную избыточность свойств до начала адаптации (т.е. ОВС > ОТФС).

В ходе адаптации данной системы к конкретному функциональному запросу под воздействием ее внутренней детерминанты внутренние свойства (потоки) системы, поддерживающие требуемую функцию, будут превращаться из интенций в экстенции, а поддерживающие, в данном случае не нужные, функциональные свойства, наоборот, - из экстенций в потенции и далее в интенции. Таким образом, в результате адаптации уменьшается избыточность свойств системы, все сильнее проявляются ее существенные для данной надсистемы свойства. Система из исходного материала, потенциально пригодного для выполнения заданной 
функции, превращается во все более совершенную субстанцию данной надсистемы, все более соответствующую запросу.

Чем глубже адаптирована система к функциональному запросу надсистемы, тем ярче проявляются ее существенные для надсистемы свойства, тем выше степень сформированности её сущности, тем меньше избыточность её свойств. Система, у которой область возможных состояний в результате адаптации к запросу надсистемы, не просто покрывает, а максимально близка к области требуемых функциональных состояний, называется оптимально адаптированной или совершенной $[7,8]$. Такие системы представляют собой четко сформированные, ярко проявляющиеся, вполне устойчивые явления определенной сущности, мера системности $\left(\boldsymbol{M}_{s}=\right.$ ОТФС/ОВС) которых близка к 1.

\section{2. Формализованное описание процесса пассивной адаптации}

Представленное содержательное системное понимание процесса адаптации можно формализовать следующим образом.

Системно-объектный подход уточняет упомянутое выше содержательное определение системы, представляя систему как триединую конструкцию «Узел-Функция-Объект», в которой узел - перекресток входных и выходных связей/потоков системы, функция - процесс преобразования входных потоков в выходные, объект - совокупность субстанциальных характеристик системы $[6,8]$. Такое представление системы позволяет использовать для формализации системно-объектного подхода исчисление объектов Абади-Кардели [9]. В результате система $\mathbf{s}$ представляется как специальный объект данного исчисления (называемый нами «узловым объектом»), состоящий из полей и методов [10]:

$$
\text { s = [(Ls?, Ls!); fs(Ls?)Ls!; (Os?, Os!, Osf)]. }
$$

Здесь (Ls? $\cup$ Ls!) $\leftrightarrow$ us - узел, который формально представляет собой поля узлового объекта для описания объектов еще одного специального вида (называемых нами «потоковыми объектами» [10]), соответствующих множеству функциональных связей данной системы; Ls? - множество входящих интерфейсных потоковых объектов, соответствующих входящим связям системы $\mathbf{s}, \mathbf{L s}$ ! множество исходящих интерфейсных потоковых объектов, соответствующих выходящим связям системы s, Ls $? \subset \mathbf{L}$ и $\mathbf{L s} ! \subset \mathbf{L}$, т.е. относятся к множеству всех связей $\mathbf{L}$ (потоковых объектов).
fs(Ls?)Ls! (fs: Ls? $\rightarrow$ Ls!) - функция, которая формально представляет собою метод узлового объекта, описывающий функцию системы $\mathbf{s}$, т.е. процесс преобразования входящих интерфейсных потоковых объектов (входящих связей системы) Ls? в выходящие Ls!.

Os? $\cup$ Os! $\cup$ Osf $=\mathbf{O s}-$ объект, который формально представляет собой множество полей узлового объекта для описания объектных (субстанциальных) характеристик системы s. Множество полей для описания объектных характеристик системы состоит из трех подмножеств: Os? - множество полей, которое содержит интерфейсные входные характеристики узлового объекта (т.е. показатели входных связей системы s), Os! - множество полей, которое содержит интерфейсные выходные характеристики узлового объекта (т.е. показатели выходных связей системы s), Osf - множество полей, которое содержит передаточные характеристики узлового объекта (показатели внутренних связей системы s).

Упомянутое выше в определении системы обусловливание функции системы функцией надсистемы, рассматривающееся как функциональный запрос надсистемы на систему с определенной функцией (внешняя детерминанта системы), формально может быть описано полем us $\leftrightarrow$ (Ls? $\cup$ Ls!) узлового объекта, соответствующего данной системе, т.е. множеством потоковых объектов, соответствующих функциональным связям системы (Ls?, Ls!). Внешняя детерминанта системы есть причина ее возникновения, цель ее существования и главный определитель ее структурных, функциональных и субстанциальных свойств. Таким образом, внешняя детерминанта системы рассматривается в качестве универсального системообразующего фактора. Функционирование же системы под влиянием внешней детерминанты, являющееся ее внутренней детерминантой, так как \которое \непосредственно определяет ее внутренние свойства (структурные, функциональные и субстанциальные свойства подсистем), формально может быть описано методом fs узлового объекта, соответствующего данной системе, т.е. в следующем виде: fs(Ls?) Ls! (fs: Ls? $\rightarrow$ Ls!). Кроме того, функционирование системы в соответствии с внешней детерминантой устанавливает между системой и надсистемой отношение поддержания функциональной способности более целого. Таким образом, можно уточнить формальное выражение для принятого определения системы следующим образом:

$$
\begin{aligned}
\mathbf{s}=[\mathbf{u s} \Rightarrow \mathbf{f s} & \Rightarrow \text { Os] и } \mathbf{s}=[(\text { Ls?, Ls!) } \Rightarrow \mathbf{f s}(L s ?) L s ! \\
& \Rightarrow \text { (Os?, Os!, Osf)]. }
\end{aligned}
$$


C учетом упомянутых выше обозначений адаптация системы к запросу надсистемы представляет собой процесс приближения fs(Ls?)Ls! (т.е. внутренней детерминанты системы) к (Ls? $\cup$ Ls!) $\leftrightarrow$ us (т.е. к ее внешней детерминанте). Или, по другому, адаптация представляет собой процесс приближения текущего функционирования системы к требуемому, задаваемому узлом системы, т.е. $\mathbf{f s}_{\text {тек }} \rightarrow \mathbf{f s} \leftrightarrow \mathbf{u s}$. Полностью адаптированной считается система, у которой внутренняя детерминанта полностью соответствует внешней, т.е. fs $\leftrightarrow$ us. Такая система функционирует в полном соответствии с функциональным запросом надсистемы.

Узел us задает область определения функции $\mathbf{f s}(\mathbf{D f s}=\mathbf{L s} ?)$ и область значений функции fs $(\mathbf{E f s}=\mathbf{L s}$ !) системы s. Конкретные характеристики преобразования/отображения fs: Ls? $\rightarrow \mathbf{L s}$ ! определяются объектными (субстанциальными) свойствами Os системы s, задающими конкретные параметры входных потоков (Os?), параметры внутренних связей (Osf), обеспечивающих выполнение процесса fs, и параметры выходных потоков (Os!). Следовательно, приближение $\mathbf{f s}_{\text {тек }}$ к fs обусловлено соответствующим изменением $\mathbf{O s}=\mathbf{O s} ? \cup \mathbf{O s} ! \cup \mathbf{O s f}$, т.е. $\mathbf{f s}_{\text {тек }}=\varphi(\mathbf{O s}(\mathbf{t}))$. T.е. процесс, так называемой пассивной адаптации в рамках системно-объектного подхода следует рассматривать в целом как процесс изменения объектных характеристик системы, приводящий $\mathbf{f s}_{\text {тек }}$ в соответствие с fs $\leftrightarrow$ us.

\section{3. Проявление общесистемных}

\section{закономерностей в процессе адаптации}

Если процесс адаптации является общесистемным, то в нем не могут не проявляться хотя бы некоторые известные общесистемные принципы и закономерности. Рассмотрим такие закономерности, использую их определения, представленные в работах [11 и 12].

После возникновения функционального запроса на систему с определенной функцией исходный материал для формирования системы, в соответствии со сказанным выше, должен выбираться, очевидно, с учетом действия закона необходимого разнообразия, который утверждает, что для создания системы, способной справится с решением проблемы, обладающей определенным разнообразием, необходимо обеспечить, чтобы система имела большее разнообразие возможностей, чем разнообразие решаемой проблемы. Данная общесистемная закономерность обуславливает способ выбора исходного материала, который будет адаптироваться к функциональному запросу (внешней детерминанте), таким образом, чтобы область его возможных состояний была шире области требуемых (т.е. ОВС > ОТФС).

Используя упомянутые выше термины и обозначения, проявление данного закона на начальном этапе формирования системы можно представить в виде следующего выражения:

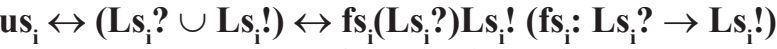

$$
\begin{aligned}
& \Rightarrow \exists \mathbf{s}_{\mathrm{i}}=\left[\left(\mathbf{L s}_{\mathrm{i}} \text { ?, Ls } \mathbf{i} \text { !); } \mathbf{f s}_{\mathrm{j}} \text { (Ls } \mathbf{s}_{\mathrm{i}} \text { ? }\right) \mathbf{L s}\right. \text { ! } \\
& \left(\mathrm{fs}_{\mathrm{i}}:\left(\mathrm{Ls}_{\mathrm{j}} \text { ? } \supset \mathrm{Ls}_{\mathrm{i}} \text { ?) } \rightarrow\left(\mathrm{Ls}_{\mathrm{i}} ! \supset \mathrm{Ls}_{\mathrm{i}} !\right)\right) ;\left(\mathrm{Os}_{\mathrm{i}} \text { ? } \supset \mathrm{Os}_{\mathrm{i}}\right. \text { ?, }\right. \\
& \left.\left.\mathrm{Os}_{\mathrm{j}} ! \supset \mathrm{Os}_{\mathrm{i}} !, \mathrm{Os}_{\mathrm{j}} \mathbf{f} \supset \mathrm{Os}_{\mathrm{i}} \mathbf{f}\right)\right]
\end{aligned}
$$

В данном выражении показано, что функциональный запрос на систему $\mathbf{s}_{\mathbf{i}}$ с функцией $\left(\mathbf{f s}_{\mathbf{i}}: \mathbf{L s}_{\mathrm{i}}\right.$ ? $\rightarrow \mathbf{L s}_{\mathbf{i}}$ !) в виде вакантного узла $\mathbf{u s}_{\mathrm{i}} \leftrightarrow\left(\mathbf{L s}_{\mathbf{i}}\right.$ ? $\cup \mathbf{L s}$ ! $)$ определяет выбор исходного материала в виде системы $\mathbf{s}$, обладающей функцией $\mathbf{f} \mathbf{s}_{\mathbf{j}}$, область определения которой и область значений включают в себя области определения и значений функции $\mathbf{f s}_{\mathbf{i}}$ $\left(\mathbf{L s}_{\mathrm{j}}\right.$ ? $\supset \mathbf{L} \mathbf{s}_{\mathrm{i}}$ ? $\wedge \mathbf{L} \mathbf{s}_{\mathrm{j}}$ ! $\supset \mathbf{L} \mathbf{s}_{\mathrm{i}}$ !). В таком же соотношении находятся объектные характеристики требуемой системы $\mathbf{s}_{\mathbf{i}}$ и системы $\mathbf{s}_{\mathbf{j}}$ как исходного материала.

После начала функционирования исходного материала в ранее вакантном узле надсистемы начинается, как отмечено выше, собственно процесс адаптации этого исходного материала к функциональному запросу. И, в первую очередь, здесь действует принцип самоорганизации, обеспечивающий процесс поступательной функционализации элементов системы. Данная общесистемная закономерность обуславливает распространение требований внешней детерминанты заданной системы на все более глубокие уровни в иерархии подсистем исходного материала.

Используя упомянутые выше термины и обозначения, проявление данного принципа на начальном этапе адаптации можно представить в виде следующего выражения:

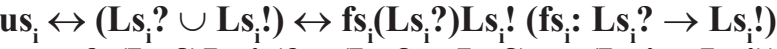

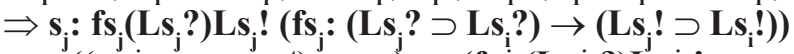

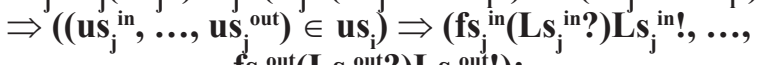

$$
\begin{aligned}
& \left.\mathbf{f s}_{\mathrm{j}}{ }^{\text {out }}\left(\mathbf{L s}_{\mathrm{j}}{ }_{\mathrm{j}} \mathrm{it} \text { ? }\right) \mathbf{L s} \mathbf{s}_{\mathrm{j}}{ }^{\text {out }} \text { ! }\right) \text { : }
\end{aligned}
$$

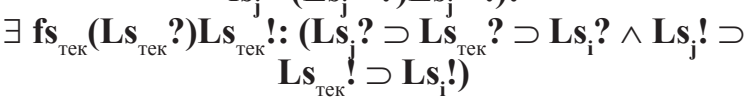

В данном выражении показано, что система $\mathbf{s}_{\mathbf{j}}$, как исходный материал, занимая вакантный узел us $\mathbf{s}_{\mathbf{i}}$ (т.е. являющаяся следствием функционального запроса us ), своим фактическим функционированием $\mathbf{f s}_{\mathrm{j}}:\left(\mathbf{L} \mathbf{s}_{\mathrm{j}}\right.$ ? $\supset \mathbf{L s}_{\mathrm{i}}$ ? $) \rightarrow\left(\mathbf{L} \mathbf{s}_{\mathrm{j}} ! \supset \mathbf{L s} \mathbf{s}_{\mathrm{i}}\right.$ !) в ранее вакантном узле надсистемы определяет функциональные требования к своим подсистемам в виде внутренних узлов $\mathbf{u s}_{\mathbf{j}}$ in, ..., us $\mathbf{j}_{\mathbf{j}}^{\text {out }}$ (для краткости названы только интерфейсные узлы, обеспечи- 
вающие функциональные связи системы $\mathbf{s}_{\mathbf{j}}$ ). При этом эти узлы, в данном случае являясь частями узла us, системы $\mathbf{s}_{\mathbf{j}}$, которые обеспечивают выполнение исходным материалом текущей функции $\mathbf{f s} \mathbf{s e к}_{\text {тек }}\left(\mathbf{L s}_{\text {тек }}\right.$ ?) $\mathbf{L} \mathbf{s}_{\text {тек}}$ !, которая занимает промежуточное положение между функцией исходного материала, т.е. системы $\mathbf{s}_{\mathbf{j}}$, и требуемой функцией заданной системы $\mathbf{s}_{\mathrm{i}}$. Указанный процесс продолжается и на уровне подсистем этих подсистем и т.д.

Можно утверждать, что в процессе адаптации работает не только принцип самоорганизации, так как последний не задает определенного механизма приближения функционирования исходного материала к требованиям функционального запроса. По-видимому, принцип самоорганизации использует возможности принципа прогрессирующей механизации, утверждающего, что части системы в ходе ее развития специализируются или становятся фиксированными по отношению к определенным функциям или механизмам. Данная общесистемная закономерность обусловливает механизм постепенного приближения в процессе адаптации фактического функционирования исходного материала к задаваемой внешней детерминантой функции требуемой системы.

Используя упомянутые выше термины и обозначения, проявление данного принципа в процессе адаптации можно представить в виде следующего выражения:

$$
\begin{aligned}
& u_{i} \leftrightarrow\left(\operatorname{Ls}_{i} ? \cup \operatorname{Ls}_{i} !\right) \Rightarrow s_{j}: f_{s_{j}}\left(\operatorname{Ls}_{i} ?\right) L s_{i} !\left(f s_{j}: \operatorname{Ls}_{i} ? \rightarrow\right.
\end{aligned}
$$

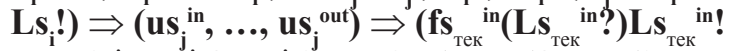

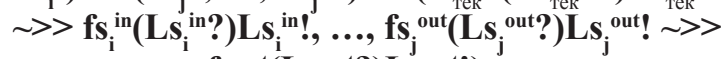

$$
\begin{aligned}
& \text { fs }_{\mathrm{i}}{ }^{\text {out }}\left(\mathrm{Ls}_{\mathrm{i}}{ }^{\text {out? }} \text { ) } \mathrm{Ls}_{\mathrm{i}}{ }^{\text {out! }}\right) \Rightarrow
\end{aligned}
$$

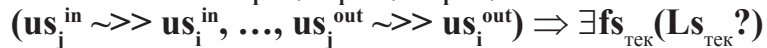

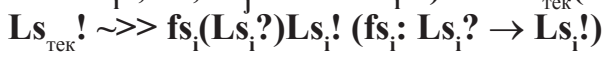

В приведенном выражении показано, что фактическое функционирование системы $\mathbf{s}_{\mathbf{j}}$ (исходного материала) в соответствии с внешней детерминантой us , т.е. преобразование требуемых потоков, а не потоков, на которые рассчитан исходный ма-

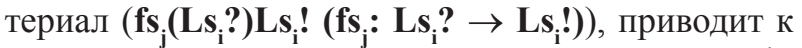
изменению требований к внутренним узлам (us ${ }_{\mathbf{j}}{ }^{\text {in }}$, ..., us ${ }_{\mathbf{j}}{ }^{\text {ut }}$ ) системы $\mathbf{s}_{\mathbf{j}}$, в результате которых их текущее функционирование, стремящееся к требуе-

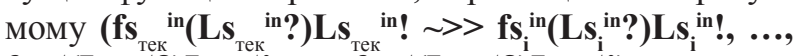

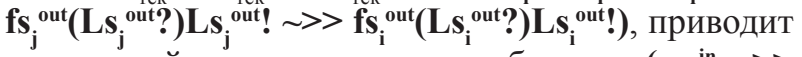
к подстройке этих узлов к требуемым (us in $>>$ $\mathbf{u s}_{\mathbf{i}}{ }^{\text {in }}, \ldots, \mathbf{u s}_{\mathbf{j}}{ }^{\text {out }} \sim>$ us $\left._{\mathbf{i}}{ }^{\text {out}}\right)$, что обеспечивает постепенное приближение общей функции исходного материала $\mathbf{s}_{\mathbf{j}}$ к требуемой внешней детерминантой

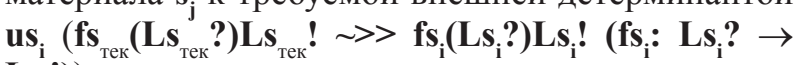
Ls $!$ )).
Принцип прогрессирующей механизации обуславливает механизм постепенного приближения в процессе адаптации фактического функционирования исходного материала к задаваемой внешней детерминантой функции требуемой системы за счет использования принципа комплиментарности (устойчивость системы достигается взаимно-дополнительными связями между её элементами в виде замкнутых контуров обратных связей), который, в свою очередь, использует принцип обратной связи (устойчивость в сложных динамических системах достигается за счёт замыкания петель обратных связей). Данное обстоятельство обусловлено тем фактом, что адаптация в конце концов должна приводить к поддержанию со стороны подсистем формируемой системы не тех функциональных связей/потоков, на которые был рассчитан исходный материал (в данном случае система $\mathbf{s}_{\mathbf{j}}$ ), a новых связей/потоков, задаваемых узлом us, т.е. потоков Ls, ? и Ls $\mathbf{s}_{\mathrm{i}}$ !, а не $\mathbf{L s}_{\mathbf{j}}$ ? и $\mathbf{L} \mathbf{s}_{\mathrm{j}}$ !. Следовательно, в системе $\mathbf{s}_{\mathbf{j}}$ должен существовать следящий механизм, который позволяет установить момент, когда связи/потоки исходного материала становятся связями/потоками требуемой системы $\mathbf{s}_{\mathbf{i}}$. Такой механизм, естественно, реализуется в соответствии с упомянутыми выше принципами.

В результате адаптации исходного материала к запросу надсистемы возникает система с заданной функцией, для которой выполняется принцип актуализации функций, утверждающий, что объект выступает как организованный лишь в том случае, если свойства его частей (элементов) проявляются как функции сохранения и развития этого объекта. Данная общесистемная закономерность характеризует систему, у которой область возможных состояний в результате адаптации к запросу надсистемы не просто покрывает, а максимально близка к области требуемых функциональных состояний и которая называется оптимально адаптированной или совершенной $[7,8]$.

Используя упомянутые выше термины и обозначения, проявление данного принципа у оптимально адаптированной системы можно представить в виде следующего выражения:

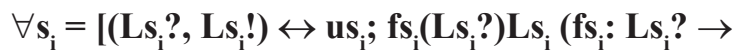

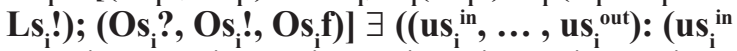

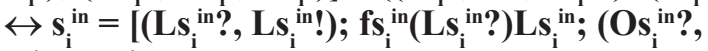

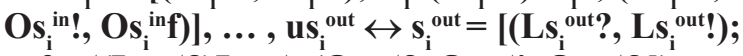

$$
\begin{aligned}
& \text { fs } \left._{i}{ }^{\text {out }}\left(\mathrm{Ls}_{\mathrm{i}}{ }^{\text {out? }} \text { ?)Ls } \mathrm{Ls}_{\mathrm{i}}{ }^{\text {out }} \text {; }\left(\mathrm{Os}_{\mathrm{i}}{ }^{\text {out? }}, \mathrm{Os}_{\mathrm{i}}{ }^{\text {out? }}, \mathrm{Os}_{\mathrm{i}}{ }^{\text {out }} \mathrm{f}\right)\right]\right) \in
\end{aligned}
$$

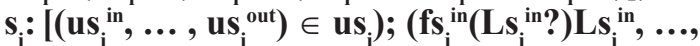

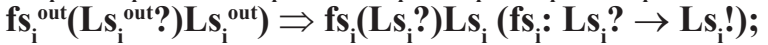

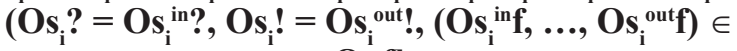

$$
\begin{aligned}
& \text { Os.f] }
\end{aligned}
$$


ФУНКЦИОНАЛЬНЫЙ ЗАПРОС НА СИСТЕМУ С ОПРЕДЕЛЕННОЙ ФУНКЦИЕЙ

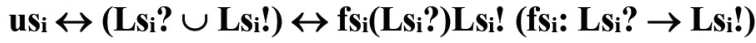

\section{ИСХОДНЫЙ МАТЕРИАЛ}

Закон необходимого разнообразия (для создания системы, способной справится с решением проблемы, обладающей определенным разнообразием, необходимо обеспечить, чтобы система имела большее разнообразие возможностей, чем разнообразие решаемой проблемы) $s_{j}=\left[\left(L s_{j} ?, L s_{j} !\right) ; f_{j}\left(L s_{j} ?\right) L s_{j} !:\left(f_{s_{j}}:\left(L s_{j} ? \supset L s_{i} ?\right) \rightarrow\left(L s_{j} ! \supset L s_{i} !\right)\right) ;\left(O s_{j} ? \supset O s_{i} ?, O s_{j} ! \supset O s_{i} !, O s_{j} f \supset O s_{i f} f\right)\right]$

\section{ПРОЦЕСС АДАПТАЦИИ}

Принцип самоорганизации (прочесс поступательной функционализации элементов системы).

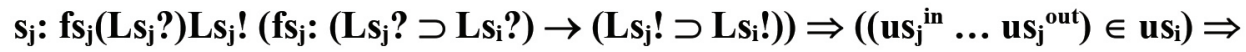

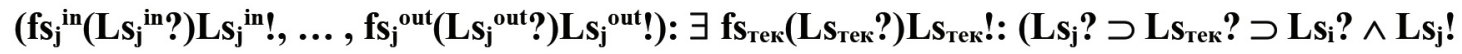

$$
\supset \mathbf{L s}_{\text {тек }} \text { ! } \supset \mathbf{L s}_{\mathbf{i}} \text { !) }
$$

Принцип прогрессирующей механизации (части системы в ходе ее развития спечиализируются или становятся фиксированными по отношению $к$ определенным функциям или механизмам)

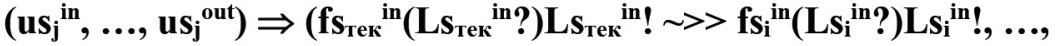

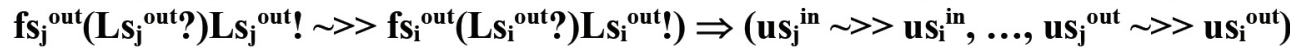

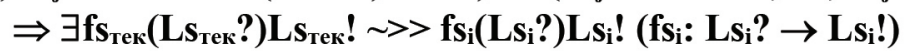

Принцип взаимно-дополнительных соотношений/комплиментарности (устойчивость системы достигается взаимно-дополнительными связями между её элементами в виде замкнутых контуров обратных связей).

Принцип обратной связи (устойчивость в сложных динамических системах достигается за счёт замыкания петель обратных связей).

СИСТЕМА С ЗАДАННОЙ ФУНКЦИЕЙ

Принцип актуализации функций (объект выступает как организованный лишь в том случае, если свойства его частей (элементов) проявляются как функции сохранения и развития этого объекта).

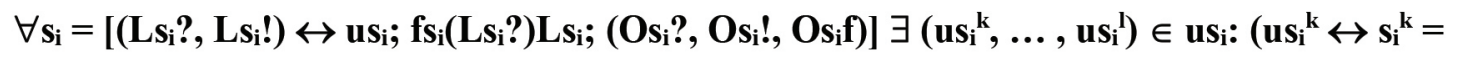

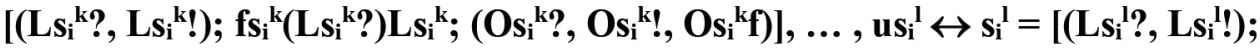

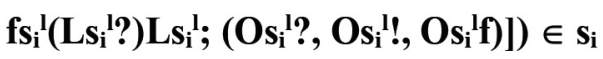

Рис. 1. Схема общесистемного процесса пассивной адаптации 
В этом выражении показано, что любая адаптированная (хорошо организованная) система $\mathbf{s}_{\mathbf{i}}$ имеет в своем составе подсистемы $\left(\mathbf{s}_{\mathbf{i}}{ }^{\text {in }}, \ldots, \mathbf{s}_{\mathbf{i}}{ }^{\text {out }}\right)$, которые в совокупности своим функционированием обеспечивают систему $\mathbf{s}_{\mathrm{i}}$.

Представленное выше содержательное и формальное описание проявления конкретных общесистемных закономерностей в процессе адаптации системы к функциональному запросу надсистемы позволяет описать в тех же терминах весь процесс пассивной адаптации, схематически представленный на рисунке.

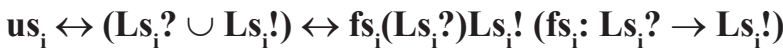

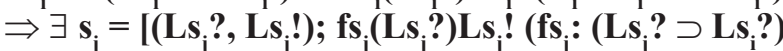

$$
\begin{aligned}
& \left.\rightarrow\left(\mathrm{Ls}_{\mathrm{j}} ! \supset \mathrm{Ls}_{\mathrm{i}} !\right)\right) \text {; }\left(\mathrm{Os}_{\mathrm{j}} \text { ? } \supset \mathrm{Os}_{\mathrm{i}} \text { ?, Os } \mathrm{s}_{\mathrm{j}} ! \supset \mathrm{Os}_{\mathrm{i}} !, \mathrm{Os}_{\mathrm{j}} \mathrm{f} \supset\right. \\
& \left.\left.\mathrm{Os}_{\mathbf{i}} \mathbf{f}\right)\right] \Rightarrow\left(\left(\mathbf{u s}_{\mathbf{j}}{ }^{\text {in }}, \ldots, \mathbf{u s}_{\mathbf{j}}{ }^{\text {out }}\right) \in \mathbf{u s}_{\mathbf{i}}\right) \Rightarrow
\end{aligned}
$$

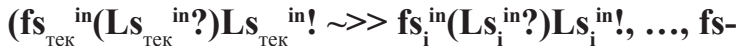

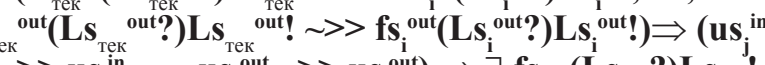

$$
\begin{aligned}
& \left.\stackrel{\text { тек }}{\sim} \text { us }_{\mathrm{i}}{ }^{\text {in }}, \ldots . \text { us }_{\mathrm{j}}{ }^{\text {out }} \sim>\text { us }_{\mathbf{i}}{ }^{\text {out }}\right) \Rightarrow \exists \mathbf{f s}_{\text {тек }}\left(\mathbf{L s}_{\text {тек }} \text { ?) }\right) \mathbf{L s}_{\text {тек }} \text { ! }
\end{aligned}
$$

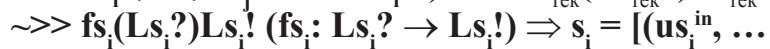

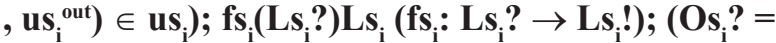

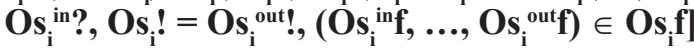

Схематически процесс пассивной адаптации самонастраивающейся системы изображен на рис. 1.

В данном выражении показано, что функциональный запрос надсистемы в виде вакантного узла $\mathbf{u s} \mathbf{s}_{\mathbf{i}} \leftrightarrow\left(\mathbf{L s}_{\mathbf{i}} \mathbf{?} \cup \mathbf{L s}_{\mathbf{i}}\right.$ !) на систему $\mathbf{s}_{\mathbf{i}}$ с функцией $\mathbf{f s}_{\mathbf{i}}: \mathbf{L s} \mathbf{s}_{\mathbf{i}}$ ? $\rightarrow \mathbf{L s}_{\mathbf{i}}$ ! приводит к втягиванию в данный узел (существованию в данном узле) исходного материала (системы $\left.\mathbf{s}_{\mathbf{j}}\right)$, у которого $\mathbf{f s}_{\mathrm{j}}:\left(\mathbf{L s}_{\mathrm{j}}\right.$ ? $\supset \mathbf{L s}_{\mathrm{i}}$ ?) $\rightarrow$

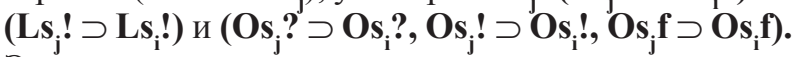
Это, в свою очередь, приводит к тому, что внутренние подсистемы системы $\mathbf{s}_{\mathbf{j}}$, соответствующие узлам этой системы, являющимся частями узла надсистемы us, начинают приводить свое функционирование к тому функционированию, которое должны иметь подсистемы заданной системы. При этом узлы исходного материала постепенно становятся требуемыми, что приводит к изменению общей функции системы $\mathbf{s}$, и таким образом из исходного материала образуется система $\mathbf{s}_{\mathrm{i}}$., удовлетворяющая требованиям надсистемы. Процесс адаптации завершается.

\section{Заключение}

Техническая творческая деятельность человека часто связана с адаптацией. Например, проектирование технических, а также информационных систем, по сути дела, есть итерационный процесс адаптации результата проектирования к требованиям технического задания. Внедрение технических и информационных систем также представляет собой процесс адаптации к условиям эксплуатации таких систем. Системное понимание механизмов адаптации позволяет уточнить необходимые в этих видах деятельности процедуры и избежать весьма вероятных ошибок.

Проведенный анализ показывает, что процесс адаптации можно конструктивно описать средствами системно-объектного подхода. Полученный результат в виде схемы процесса адаптации уточняет системное понимание данного процесса и обосновывает тот факт, что его механизм основан на взаимодействии конкретных общесистемных закономерностей. Учет этих закономерностей в процессе адаптации системы является и теоретически, и практически полезным.

\section{Литература}

1. Волкова В.Н. Теория систем и системный анализ в управлении организациями: Справочник / Под ред. В.Н. Волковой и А.А. Емельянова. М.: Финансы и статистика, 2006. - 848c.

2. Растригин Л.А. Адаптация сложных систем. Рига: Зинатне, 1981. - 375c.

3. Сурмин Ю.П. Теория систем и системный анализ. - К.: МАУП, 2003. - 368c.

4. Артюхов B.B. Общая теория систем: Самоорганизация, устойчивость, разнообразие. - М.: Книжный дом ЛИБРОКОМ, 2012. - 224c.

5. Фрадков А.Л. Кибернетическая физика: принципы и примеры. - С.-Петербург: Наука, 2003. $-208 \mathrm{c}$.

6. Маторин С.И., Попов А.С., Маторин В.С. Моделирование организационных систем в свете нового подхода «Узел-Функция-Объект» // Научно-техническая информация. Сер.2. - 2005. - N1. - C. 1-8.

7. Мельников Г.П. Системология и языковые аспекты кибернетики. - М.: Сов.радио, 1978. - 368c.

8. Маторин С.И., Зимовеи О.А. Общая теория систем. - Белгород: Изд-во БУКЭП, 2014. - 178 с.

9. Abadi Martin and Luca Cardelli A Theory of Objects. - New York: Springer-Verlag. - 1996. $397 \mathrm{p}$.

10. Жихарев А.Г., Маторин С.И., Зайцзева Н.О. Системно-объектный инструментарий для имитационного моделирования технологических процессов и транспортных потоков // Искусственный интеллект и принятие решений. - 2015. - №4. - С. 95-103.

11. Маторин С.И., Зимовец О.А., Жихарев А.Г. Общесистемные принципы в терминах системно-объектного подхода «Узел-Функция-Объ- 
ект» // Труды ИСА РАН. - 2016. - №1 - Том 66. - С. 10-17.

12. Маторин С.И., Жихарев А.Г. Учет общесистемных закономерностей при системно-объектном моделировании организационных знаний // Искусственный интеллект и принятие решений. - 2018 - №3 - С. 115-126.

Маторин Сергей Игоревич. Заместитель генерального директора по науке и инновациям ЗАО «СофтКоннект», г. Белгород, ул. Студенческая, д.19, кор.1. Доктор технических наук, профессор. Количество печатных работ: более 200 (в т.ч. 5 монографий). Область научных интересов: системный подход, теория систем, системный анализ, CASE-технология, управление знаниями, бизнес-моделирование. E-mail: matorin@softconnect.ru

Жихарев Александр Геннадиевич. Доцент кафедры информационных систем Национального исследовательского университета «Белгородский государственный университет», г. Белгород, ул. Победы, д.85. Кандидат технических наук. Количество печатных работ: 90. Область научных интересов: системный анализ, управление знаниями, бизнес-моделирование, CASE-технология. E-mail: zhikharev@bsu.edu.ru.

\title{
Patterns of adapting systems to functional requirements
}

\author{
S.I. MatorinI, A.G. ZhikharevII \\ I "SoftConnect" CJSC, Belgorod, Russia \\ II National Research University "Belgorod State University”, Belgorod, Russia
}

\begin{abstract}
Annotation. The paper discusses the possibility of analyzing the adaptation of systems to functional requirements using the system-object approach "Unit-Function-Object". A substantial description of the adaptation process in terms of a system-object approach is given. For a formal description of the adaptation process, the apparatus for calculating the objects of Abadi-Kardeli was used. The connection between adaptation and system-wide regularities is shown. A scheme of passive adaptation of self-tuning systems has been developed, taking into account these patterns.
\end{abstract}

Keywords: system-object approach "Unit-Function-Object", adaptation, external determinant; inner determinant; system-wide patterns.

DOI: $10.14357 / 20790279200105$

\section{References}

1. Volkova V.N. 2006. Teoriya sistem i sistemnyj analiz v upravlenii organizaciyami: Spravochnik [System Theory and Systems Analysis in the Management of Organizations: Handbook]. M.: Finansy i statistika. 848.

2. Rastrigin L.A. 1981. Adaptacija slozhnyh sistem [Adaptation of complex systems]. Riga: Zinatne. 375.

3. Surmin Ju.P. 2003. Teorija sistem i sistemnyj analiz [Systems theory and systems analysis]. K.: MAUP. 368.

4. Artjuhov V.V. 2012. Obshhaja teorija sistem: Samoorganizacija, ustojchivost', raznoobrazie
[General systems theory: Self-organization, sustainability, diversity]. M.: Knizhnyj dom LIBROKOM. 224.

5. Fradkov A.L. 2003. Kiberneticheskaja fizika: principy i primery [Cybernetic physics: principles and examples]. S.-Peterburg: Nauka. 208.

6. Matorin S.I., Popov A.S., Matorin V.S. 2005. Organization simulation technology in the light of a new Unit-Function-Object approach. Automatic Document and Mathematical Linguistics. New York: Allerton Press, Inc. 1(39): 1-8.

7. Mel'nikov G.P. 1978. Sistemologiya i yazykovyye aspekty kibernetiki [Systemology and language aspects of cybernetics]. M.: Sov. Radio. 368. 
8. Matorin S.I., Zimovec O.A. 2014. Obshhaja teorija sistem [General theory of systems]. Belgorod: Izdvo BUKJeP. 178.

9. Abadi Martin and Luca Cardelli 1996. A Theory of Objects. New York: Springer-Verlag. 397.

10. Zhiharev A.G., Matorin S.I., Zajceva N.O. 2015. Sistemno-ob\#ektnyj instrumentarij dlja imitacionnogo modelirovanija tehnologicheskih processov i transportnyh potokov [System-object tools for simulation of technological processes and traffic flows]. Iskusstvennyj intellekt i prinjatie reshenij [Artificial Intelligence and Decision Making]. 4: 95-103.

11. Matorin S.I., Zimovec O.A., ZHiharev A.G. 2016. Obshhesistemnye principy $\mathrm{v}$ terminah sistemno- ob\#ektnogo podhoda «Uzel-Funkcija-Ob\#ekt» [System-wide principles in terms of the systemobject approach «Unit-Function-Object»]. Trudy ISA RAN [Proceedings of the Institute of System Analysis of the Russian Academy of Sciences]. 1(66): 10-17.

12. Matorin S.I., ZHiharev A.G. 2018. Uchet obshchesistemnyh zakonomernostej pri sistemnoob"ektnom modelirovanii organizacionnyh znanij [Accounting for system-wide laws in system-object modeling of organizational knowledge]. Iskusstvennyj intellekt i prinyatie reshenij [Artificial Intelligence and Decision Making]. 3: 115-126.

Matorin Sergey Igorevich. Deputy Director General for Science and Innovations of "SoftConnect" CJSC, Belgorod, Studencheskaya Street, 19, building 1. Doctor of Technical Sciences, Professor. Number of publications: more than 200 (including 5 monographs). Area of scientific interests: system approach, system theory, system analysis, CASE-technology, knowledge management, business modeling.

Zhikharev Alexander Gennadievich. Associate Professor, Chair of Information Systems, National Research University "Belgorod State University", Belgorod, Victory Street, 85. Candidate of Technical Sciences. Number of publications: 90 . Area of scientific interests: systems analysis, knowledge management, business modeling, CASE-technology. 